\title{
Prestige and content biases together shape the cultural transmission of narratives
}

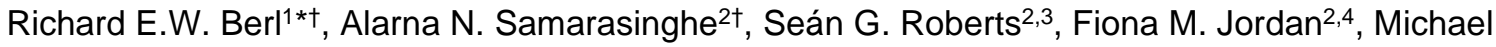 \\ C. Gavin ${ }^{1,4}$ \\ ${ }^{1}$ Department of Human Dimensions of Natural Resources, Colorado State University, Fort \\ Collins, CO 80523-1480 \\ 2 Department of Anthropology and Archaeology, University of Bristol, Bristol, United Kingdom \\ ${ }^{3}$ School of English, Communication and Philosophy, Cardiff University, Cardiff, United Kingdom \\ ${ }^{4}$ Max Planck Institute for the Science of Human History, Jena, Germany \\ * Corresponding author: Richard E.W. Berl, rewberl@colostate.edu, 970-491-6591
}

${ }^{\dagger}$ R.E.W.B. and A.N.S. contributed equally to this work and are co-first authors.

R.E.W.B., A.N.S., F.M.J., and M.C.G. designed research; R.E.W.B. and A.N.S. performed research; S.G.R. contributed new analytic tools, R.E.W.B., A.N.S., and S.G.R. analyzed data; and R.E.W.B., A.N.S., S.G.R, F.M.J., and M.C.G. wrote the paper.

\begin{abstract}
Context-based cultural transmission biases such as prestige are thought to have been a primary driver in shaping the dynamics of human cultural evolution. However, few empirical studies have measured the importance of prestige relative to other effects, such as the content biases present within transmitted information. Here, we report the findings of an experimental transmission study designed to compare the simultaneous effects of a high- or low-prestige model with the presence of content containing social, survival, emotional, moral, rational, or counterintuitive information. Results from multimodel inference reveal that prestige is a significant factor in determining salience and recall, but that several content biases, specifically social, survival, negative emotional, and biological counterintuitive information, are significantly more influential. Further, we find evidence that prestige serves as a conditional learning strategy when no content cues are available. Our results demonstrate that content biases serve a vital and underappreciated role in cultural transmission.
\end{abstract}

\section{Introduction}

Storytelling is a powerful and universal tool that humans use to know and understand the world 11,2 , to preserve history and traditional knowledge ${ }^{3,4}$, to educate ${ }^{5,6}$, to persuade ${ }^{7,8}$, and to heal $\left.\right|^{9,10}$.

Stories encode complex cultural and ecological information, and have the capability to endure for at least 7,000 years ${ }^{11,12}$, and possibly much longer ${ }^{13}$. In addition, skilled storytelling may increase an individual's reproductive fitness ${ }^{14,15}$ and social value, as well as promoting cooperation within groups ${ }^{15}$. Stories can be an efficient and effective vector for information transfer ${ }^{16}$, and an established body of literature in the interdisciplinary field of cultural evolution suggests that the success or failure of a story and its component parts are determined by the mechanisms of biased cultural transmission ${ }^{17-22}$.

The extent to which cultural selection, by way of biased transmission, is the primary factor responsible for cultural change is a central and enduring debate within cultural evolution ${ }^{23-}$ 
26. Cultural selection theory argues that cultural diversity is largely shaped by direct and indirect cognitive biases that unconsciously drive the selection of cultural variants over successive transmission events ${ }^{27-29}$. In the absence of these transmission biases, cultural learning is unlikely to be more advantageous than individual learning ${ }^{30-32}$. In this study, we provide a realistic approach to studying cultural change through investigating the relative effects of an array of competing biases within the transmission of narrative stories. This framework allows us to gain a better understanding of the microevolutionary processes that have shaped and continue to shape human culture.

Despite the critical role that transmission biases appear to play in driving cultural evolution, critical gaps exist in our understanding of the relative strengths of these biases ${ }^{25,33-35}$. In particular, prior experimental studies have tended to focus on individual biases, yet multiple biases are always present simultaneously ${ }^{19,21,36-38}$. Narratives are especially dense in information that contains a number of proposed content-based or "direct" biases ${ }^{28}$ that have been shown to aid in the salience and retention of information ${ }^{20,39-42}$.

Content biases influence transmission through properties of the information itself that make it more appealing and memorable ${ }^{28}$. These preferences for certain types of information can vary between individuals and across cultures, but some have been seen to be remarkably consistent ${ }^{43}$. Here, we conduct the first simultaneous test of the relative effects of the most frequently cited content biases from the cultural evolution literature. This includes content linked to the following six types of information: (I) social, either in the sense of everyday basic social interaction or of "gossip" about third parties ${ }^{20,21}$; (ii) survival, for environmental contexts relevant to individual fitness 21,41,44; (iii) emotional, that elicits strong positive or negative responses such as disgust ${ }^{19,40,45,46}$; (iv) moral, regarding acceptable behavior and social norms ${ }^{19,47}$, which has not been previously studied explicitly using transmission experiments; $(v)$ rational, describing causeand-effect connections ${ }^{48}$; and ( $\left.v i\right)$ counterintuintuive, which defies ontological expectations in biological, physical, mental, and other domains ${ }^{39,49}$. Additionally, counterintuitive information can influence transmission in different ways: by themselves, counterintuitive elements can be more salient than other types of information 39 ; or, collectively, a minority of counterintuitive elements can lead to a minimally counterintuitive ("MCl") bias that enhances overall recollection of a story ${ }^{22,50}$. We crafted the narratives used in this study to resemble real-world creation stories in both form and the aforementioned types of content biases (see Methods). Real-world creation stories have evolved over many generations of transmission and selection, and therefore tend to contain biased content at high frequencies.

Beyond the types of information included in a story, learners are also sensitive to the identity and reputation of the storyteller. These transmission biases are referred to as contextbased biases, and include model-based or "indirect" 28 biases such as prestige ${ }^{51}$, success ${ }^{52}$, and similarity ${ }^{53,54}$, and frequency-dependent conformity and anti-conformity biases ${ }^{55}$. In this study, we specifically examine prestige bias, which involves a preference to learn from individuals of high social position, reputation, and knowledge ${ }^{56}$. Prestige bias is one of the most commonly cited transmission biases ${ }^{35}$, and has been implicated as one of the predominant forces in cultural change ${ }^{51,57,58}$. However, the limited empirical work to date has shown mixed support regarding the extent to which the prestige of a model actually affects the adoption of a particular cultural variant or behavior ${ }^{38,59-61}$ (see ${ }^{35}$ for a recent general review on the topic).

We use regional accents of speech as an experimental cue for prestige information. As has been established within the field of sociolinguistics ${ }^{62-66}$, and verified by two of our previous studies $^{56,67}$, listeners perceive accents as strong indicators of prestige. Accents are hard-to-fake signals ${ }^{68}$ and tend to be stable over time. Therefore, some varieties become associated with membership in high-status groups ${ }^{63,69-71}$. These perceptions of accent are consistent with how prestige is understood in cultural evolution studies. Accent thus provides a methodological alternative to the use of attention, gaze, or group consensus to represent prestige, which 
potentially suffer from flaws tied to the ambiguity and context dependency of these signals ${ }^{26,72-74}$. In our experiment, we present stories aurally and ask for oral recall rather than written responses in order to limit the number of distinct cognitive domains involved.

Humans are highly attuned to the biases present in the information we consume and to the identities of potential cultural models that hold that information. Here, we address multiple gaps in the literature by explicitly quantifying learners' recall of multiple distinct types of content, transmitted by speakers with varying levels of prestige. By testing content and context biases together in the experimental transmission of a narrative, we can examine the relative effects of a large suite of biases: biases that theory suggests shape the spread of information and the evolution of human culture.

\section{Results}

Participants showed preferential recall of biased information. Of the 87,421 narrative propositions we presented in total, participants recalled 12,505 (14.3\%) (Supplementary Table 1). We found a significant difference between the proportions of content types presented and the proportions of content types recalled (two-sided permutation test of independence: $z=-2.037, p=$ 0.042 ), showing that participants recalled some types of biased information more frequently than other types, including unbiased information (i.e. propositions that did not contain any of the examined content biases; Fig. 1).

Recall for each type of content bias ranged from a mean of $6.6 \%$ of the propositions presented (moral) to $33.9 \%$ (biological counterintuitive). In general, we observed small but nonsignificant differences in the recall of content biases in high- versus low-prestige speaker conditions (Fig. 2). However, corrected pairwise comparisons of proportions (Supplementary Table 2) showed that prestige had a significant impact on the recall of unbiased information $(p<$ $0.001)$ and basic social information $(p=0.001)$. Additionally, participants recalled unbiased information significantly less often than biased information under the same prestige condition, except for positive emotional, moral, rational, and physical and mental counterintuitive information. Of these, positive emotional, moral, and mental counterintuitive information were recalled significantly less frequently than unbiased information.

Content biases were more influential than prestige bias. To explain the variance in recall of specific propositions, we fit a total of 58 proposed models using maximum likelihood estimation (Supplementary Table 3). Models included different combinations of variables for story-based effects, the high or low prestige condition, the presence or absence of each content bias, and participant demographics (see Methods for a full list). Eleven of the best-fitting models had a resulting $\triangle \mathrm{AIC}$ score $<2$, indicating no single "best" model exists. The majority of best-fitting models included variables for story presentation order, for prestige, social, survival, negative emotional, and counterintuitive biases, and for gender and working memory (Supplementary Table 4).

Our results (Fig. 3; Supplementary Table 5) show that the transmission biases with the greatest effect on recall were, in descending order: counterintuitive (but only for biological violations), negative emotional, social, survival, and prestige. All other biases had negligible effects according to their model-averaged coefficients and confidence intervals and their relative variable importance values. Though we did find a significant effect for prestige, it was the weakest of the transmission biases, with an odds ratio of $1.164(95 \% \mathrm{Cl}[1.113,1.217])$ compared to the next lowest, survival, with $1.858(95 \% \mathrm{Cl}[1.216,2.841])$ and to the strongest effect, biological counterintuitive, with $7.558(95 \% \mathrm{Cl}[3.913,14.597])$. For story effects, participants had better recall for the second story they were presented, regardless of which story it was. The placement of 
propositions within the story had no effect on recall. For demographic variables, only working memory had a significant positive effect.

Transmission biases explain little variance in recall. The set of best-fitting models $(\triangle \mathrm{AIC}<2)$ had relatively high mean conditional $R^{2} G L M M$ values at $0.524(S D<0.001)$, but a lower marginal $R^{2}{ }_{G L M M}$ at $0.106(S D=0.002)$. The difference between the two values represents the proportion of the variance explained by the random effects of the model, which were the participant ID (i.e. individual differences) and proposition number. Comparisons of the lowest-AIC model with ones excluding either random effect using likelihood-ratio tests were both significant (participantID $X^{2}[1]$ $=6526.1$; proposition $X^{2}[1]=9728.2$; both $p<<0.001$ ), indicating that the individual participant and proposition effects were both influential. These results tell us that there is a great deal of variance in our responses that is not accounted for by the transmission biases and other fixed effects included in the models. Further, they indicate that this unexplained variance exists both among the participants and within the content of the stories.

\section{Discussion}

Prestige bias has a minor effect on transmission. We found significant positive effects for prestige, social, survival, negative emotional, and biological counterintuitive biases on recall (see Fig. 3; Supplementary Table 5). Prestige-biased transmission has been prominent in the cultural evolution literature $28,35,36,51,75-79$. However, prestige bias as proxied by accent had the smallest effect on transmission, increasing the likelihood of a proposition's recall by only $15 \%$. One possible explanation for the secondary importance of prestige concerns the nature of the narratives transmitted. Transmission biases can lead to the development of group markers and ingroup cooperation ${ }^{53,80,81}$, and creation stories are representative of a shared group identity ${ }^{15}$. We propose that if the audience does not perceive some cultural relationship between themselves and the storyteller or narrative, prestige may be a less pertinent cue for social learning. Prestige often exists as an ingroup hierarchy with less relevance to outgroup individuals 58,82 .

Assuming that shared identity could be a factor mediating the efficacy of prestige bias-in effect, a similarity bias ${ }^{53}$ - we examined links between participant and storyteller demographics. We would predict from this argument that participants should better recall a narrative read by a speaker whose accent they could personally identify with. However, our results show no effect on recall from matching participants' childhood location with the region of the low-prestige speaker's accent ("childhood town low prestige," see Fig. 3). We included other potential effects of similarity bias through the standardization of speaker demographics and the inclusion of participants' demographics in the models (see Methods). However, we found no significant associations between recall and similarity of participants' identities with those of the speakers.

Prestige is unconsciously employed as a secondary bias. Another potential explanation for the low importance of prestige in determining recall is that participants may adjust their social learning strategies depending on which biases are present in different parts of the narrative ${ }^{33,37,83}$. When content biases were present, prestige had less relative influence on recall, but participants tended to recall unbiased propositions more frequently when the narrative was told by a speaker with a high-prestige accent (Fig. 2).

The finding that prestige takes a secondary role to content supports the conclusion of the only other experimental study we know to have compared prestige and content ${ }^{38}$. In that study, the authors found that the effects of prestige were minimal compared to content effects (in the form of "inspiration" or general likability rather than specific biases) when rating their preference 
for quotations from famous or unknown authors. We suggest that, together, the results of the previous study and our own demonstrate the importance of content biases in directing cultural transmission. These content cues can be more nuanced than general context-based copying rules such as prestige, but our results show that content biases can take a primary role over context. Future studies can seek to understand how the relative importance of content versus context biases may vary across different sociocultural contexts and the potential interactive effects between different forms of biases (e.g., one character feeding another may encode both social and survival information, and be more or less salient than either type of content individually).

Content biases have distinct effects. As previously noted, we found that the effects of content types on information transmission varied widely (Fig. 3). Although we might have expected a greater attention to "gossip" over basic social interactions ${ }^{20}$, the lack of a significant difference between the two in our results (Fig. 2) could be due to variation in how we operationalized the concept of gossip. In this study, gossip was qualified by the presence of third parties in social interactions and not by the subjective intensity of interaction as has been done previously ${ }^{20}$. Furthermore, as entire narratives have been ascribed as gossip in previous work ${ }^{20}$, any recall in those studies was attributed to this bias, whereas we coded specific propositions with social interaction as either basic or gossip. The advantageous impact of social "gossip" on transmission also may have been tempered by the cognitive load of processing multiple levels of theory of mind in these interactions 84,85 .

Our results also support multiple prior empirical studies that found strong positive effects on transmission for survival information ${ }^{21,41,44,86}$, and for negative emotional information but not positive emotional information ${ }^{18,19,87}$. Indeed, negative emotional information was found to be one of the most powerful biases in our stories (Fig. 2). As negative information arouses strong emotional responses such as fear, disgust, and anger, some theorize that humans evolved broad cognitive domains receptive to negative information as a survival response to predators and toxic food sources ${ }^{88-92}$, which may explain why both survival and negative emotional information are particularly salient.

We did not find evidence to support effects from moral, rational, or most counterintuitive information on transmission. Moral and mental counterintuitive information (as well as positive emotional, above) were actually recalled less often than unbiased information (Fig. 2), though not enough to lead to negative odds ratios when accounting for other variables (Fig. 3). However, there have been few prior tests of these biases within an experimental transmission paradigm. For instance, previous evidence of a bias for "rational" or causal information in this context has been anecdotal ${ }^{93}$, though related work has focused on causal reasoning and imitation ${ }^{94-97}$. The transmission of rational information relies upon the retention of a predicate, hence, rational bias may affect the recall of surrounding information but may not be reliably recorded. Further, we defined successful transmission of rational information as requiring the retention of the subordinating conjunction ("because," "so that," "when," etc.; the proposition coded as having rational content), which may explain the lack of an effect. Hence, rational bias may have had a proximity effect on the recall of surrounding information, without being recalled itself, that was not detected by our present analyses.

For moral information, according to social norm theory, individuals should be expected to retain and transmit moral information depending, firstly, on the strength of the social norm and, secondly, on the extent to which they identify with the social group to which it applies ${ }^{98,99}$. That participants did not recall moral information is less surprising if they recognized that the creation stories did not describe their own society's origins or rules of accepted behavior. 
Narrative structural features may aid transmission. To the best of our knowledge, no existing theory addresses why particular counterintuitive domains should be recalled more frequently than others. However, our data demonstrate that biologically counterintuitive information was significantly more likely to be transmitted. This result may not necessarily be due to biased content per se, but rather could be a consequence of narrative construction. Many of the biological counterintuitive propositions in our stories were repetitive in structure (for example, in the "Muki" story, spiders were transformed into other animals four times in sequence), and recollection may be affected by what Jakobson ${ }^{100}$ called the "poetic function" of language ${ }^{101}$, or the artistic quality of the message itself. In our study design, we credit a causal role to linguistic factors in social learning through our use of accent-based prestige; however, narrative theory itself remains a rich and largely untapped resource in cultural evolutionary accounts of information transmission ${ }^{102}$.

For stories to be impactful, the content must engage the audience ${ }^{103-105}$ and compete for space in working memory ${ }^{106-109}$. To this end, stories (and their tellers) employ a suite of features to enhance their salience, including elements that evoke emotional arousal ${ }^{110-113}$ and the use of familiar narrative devices such as rich encoding and repetition ${ }^{114,115}$. As such, multiple factors influence the success of story transmission and the data demonstrate that transmission biases alone do not capture the full variation.

Implications for the understanding of transmission. The overall fit of our model is high $\left(R^{2} G L M M C=0.524\right)$, but fixed effects only explain a small portion of the variation in recall $\left(R^{2} G L M M m=\right.$ $0.106)$. One possible explanation for this result is that some as-yet unidentified biases exist in the characteristics of the models or in the content of the stories, and this drives the variation in proposition transmission. However, our methodological approach included every type of content bias supported in the literature, and we could not test the remaining well-documented context biases, such as conformity bias ${ }^{116}$ and success bias ${ }^{117}$, because they do not apply to the one-toone transmission context of our experiment. In the future, if additional content biases are identified in the literature, it would be possible for researchers to re-code our data (see Repository) to test them.

Instead, the substantial explanatory power of the random effects in our models may represent the noise of individual variation. The trade-off for gaining real-world experimental validity is typically a greater amount of noise due to uncontrolled circumstances. Our methodological approach did not allow us to control the testing environments, including levels of distraction, participants' levels of attention, or participants' personal short and long-term histories. In this way, the experiment mimics real-world cultural transmission, which tends to be filled with random noise that can lead to low fidelity in one-off transmission events ${ }^{118,119}$. Much debate exists regarding the degree of transmission fidelity required for cumulative culture. Some argue that high-fidelity transmission is required ${ }^{120-124}$, while others counter that low-fidelity transmission is sufficient ${ }^{125-131}$ and that weak biases can be amplified over repeated rounds of transmission to create strong universal patterns ${ }^{119,132,133}$. We found that participants' responses to identical stimuli varied significantly, and transmission fidelity was often low compared to previous studies ${ }^{20}$. Participants knew they would need to retain and recite the information, but on average they recalled only $14.3 \%$ of the propositions presented $(S D=10.4 \%)$. In the context of a single-shot experimental transmission event, however, participants have no real incentive to retain information, and these stories were intentionally of considerable length and posed a substantial challenge for working memory. Furthermore, repeated exposure to a story increases comprehension ${ }^{134}$, and narratives that particularly define a group-such as creation stories-are often told multiple times ${ }^{135}$ or are collaborative, with opportunities for audience engagement that allow group members to transform and take ownership of the narrative ${ }^{136,137}$. Future work, both 
theoretical and empirical, should consider how models of transmission processes can accurately incorporate individual variation in cultural transmission and responses to content.

Our methodological and analytical framework provides a template for future tests of the simultaneous effects of context and content biases. We have performed an experimental test of the relative effects of multiple types of cultural transmission biases presented within a realistic package of narrative information, while incorporating linguistic factors that have been underutilized in the cultural evolution literature. Although we found that prestige was the least important transmission bias, it was still a significant factor in participants' choices of what information to retain and recall, especially for information lacking any internal biases. Our results suggest that the prominent role of prestige-biased transmission models in cultural evolution studies should be scrutinized more heavily and qualified by the presence or absence of other biases, which may have stronger effects under certain conditions. The experimental framework presented here sets the stage for future research to test longstanding questions in cultural evolution, such as: which biases are necessary or sufficient for the development of cumulative culture $^{120}$, which conditions cause learners to favor one type of bias over another ${ }^{83}$, whether and how the effects of different biases differ cross-culturally ${ }^{118,138-140}$, how micro-level transmission processes lead to macro-level cultural change 20,141 , and how we can identify the bias or biases responsible for a post hoc distribution of traits ${ }^{142}$. The results of this study go beyond academic discourse in cultural evolution to impact other disciplines that rely on the theory and application of communication as a means of disseminating information and motivating behavior change, including education, marketing, conservation, public health, and political science. Storytelling persists as a powerful and enduring tool, dense in cultural information, and utilized across the world to share knowledge and shape the diversity of human culture.

\section{Methods}

Story production. We selected creation stories, which often pertain to the origins of life, death, nature, and human society, as the narrative form to be used for this study because they are rich in the types of content proposed to be relevant to cultural transmission. Further, creation stories are a familiar pattern cross-culturally for the transmission of knowledge, values, and meaning, and have each individually been subject to many generations of transmission and transformation.

We undertook a survey of creation stories using ethnographic data from the electronic Human Relations Area Files (eHRAF) World Cultures database ${ }^{143}$. We conducted the survey by searching for "creation" (and its derivatives) or "origin" within texts indexed under the "mythology" subject code (\#773). We performed the search in the Probability Sample Files (PSF) subset, which is a stratified random sample of 60 cultures, each representative of a different "culture area." Our search returned 100 story extracts from 35 cultures, and from this we selected 4 texts for analysis on the basis of appropriate length ( $-300-800$ words) and being written and shared by in-group authors (rather than foreign ethnographers). The stories selected belonged to the A.chik Mande (referred to in eHRAF as "Garo"), Baganda ("Ganda"), Kainai ("Blackfoot"), and Kanaka Maoli ("Hawaiian") peoples. We also included the Genesis creation story (from the ancient Israelites), as presented in the New Revised Standard Version Bible ${ }^{144,}$ Gen. 1.1-2.3. We coded the resulting 5 ethnographic creation stories at the level of propositions (word clusters consisting of "a predicate plus a series of ordered arguments"20) for the presence of social, survival, emotional, moral, rational, and counterintuitive content biases. Definitions of these biases as used for coding are listed in the Supplementary Information (Supplementary Table 6). We carried out propositional analysis under the protocol established by Turner and Greene ${ }^{145}$.

For the experiments, we commissioned two written artificial creation stories (see Acknowledgements). We did this rather than using the ethnographic stories we sampled in order 
to avoid issues of cultural appropriation surrounding the use of stories from real societies, and to ensure that our participants would all be equally unfamiliar with the stories. We recognize that stories artificially created to satisfy controls have not naturally evolved through cultural transmission, however, this confound cannot be removed from experimental work requiring comparable stimulus material. To minimize this effect, a professional author wrote the stories, and we aimed to preserve narrative flow in subsequent story revisions. The first story, "Muki," explains how the actions of a child abandoned by its parents shaped a rugged landscape and its varieties of life-forms. The second story, "Taka \& Toro," describes two jealous seafaring siblings and their competition over the friendship of the people they created. Over many iterations, we edited the texts of these artificial creation stories at sentence- and then proposition-level to ensure the proportions of each type of biased proposition in each story matched one another, and also fell within $90 \%$ confidence intervals of the proportions seen in the coded ethnographic creation stories (Supplementary Table 7). We tuned both stories to be approximately 850 words (Muki 887, Taka \& Toro 835) and 270 propositions (Muki 265, Taka \& Toro 273) to avoid ceiling effects for recall and to be of roughly equal complexity. Readability scores (based on number of syllables per word and sentence structure) for these artificial stories were roughly equivalent and used simpler language than the ethnographic stories they were modeled after (Flesch-Kincaid grade level: Muki 4.91, Taka \& Toro 5.03, Ethnographic Mean 8.22 [90\% Cl: 6.42, 10.02]; Flesch reading ease: Muki 84.5, Taka \& Toro 81.9, Ethnographic Mean 71.24 [90\% Cl: 62.24, 80.24]). The final versions of the two artificial creation stories, along with lists of their propositions and coded biases, can be found in the Repository.

Recordings. We used language accent to index prestige, in line with findings from sociolinguistics ${ }^{63,66,146-148}$. Language attitude studies have demonstrated that non-localized "standard" accents are associated with high prestige ${ }^{149-152}$ based on ideological values ${ }^{65,153}$, although regional non-standard accents demonstrate differential prestige ${ }^{63-65,154}$. We recorded self-identified middle-aged white male speakers with high- and low-prestige accents calibrated for the participants' locations telling the two stories ("Muki" and "Taka \& Toro"). We selected the highand low-prestige accents based on the results of a previous study ${ }^{67}$. For both the UK and USA participants, Received Pronunciation ("RP") was the high-prestige accent. For the UK sample, the low-prestige accent was West Country, from South West England; and for the US sample, the low-prestige accent was Inland South, spanning the southern Appalachian, Ozark, and Ouachita mountain ranges. We standardized the recordings for volume and length (each 5 min, $19 \mathrm{~s}$ ).

For an independent assessment of accent prestige, we also recorded our speakers reading the first paragraph of the Comma Gets a Cure passage (see Acknowledgements). This passage contains words from Wells's lexical set, designed to highlight phonological variation between different accents of English ${ }^{155}$. We presented these recordings (range $35 \mathrm{~s}$ to $39 \mathrm{~s}$ ) to participants to confirm that their perceptions of the prestige of each speaker matched what was expected (see Experimental protocol, below).

Participants. We recruited UK participants on the Prolific Academic platform $(n=96)$, and US participants on Amazon Mechanical Turk $(n=100)$ using TurkPrime ${ }^{156}$. Participants were eligible to take part in this study if they: had not taken part in any previous studies by the researchers; had taken part in and had successfully completed over 95\% of at least 100 studies on Prolific Academic or over $98 \%$ of at least 5,000 tasks on Amazon Mechanical Turk; and were native English speakers. We excluded data from 33 participants due to technical recording errors or external interference (e.g. a second person contributing to the recall of a story).

Experimental protocol. The experiment was administered through a custom web browser application using the SurveyJS library (source code available at: 
https://github.com/seannyD/StoryTransmission). Participants were directed from their respective recruitment platforms to the web application on University of Bristol servers. Participants first selected their location, which determined which of the locally-calibrated accent recordings they would hear. Participants were instructed to listen once (due to expected completion rates) to a recording of the first artificial creation story and were told that they would be asked to recall the story in as much detail as possible.

After listening to a creation story, participants took part in a working memory distraction task based on the Visual Spatial Learning Test ${ }^{157}$. This task involved playing three rounds of a game in which participants had to recall symbols and their positions on a grid. For the symbols, we used the 9 most dissimilar characters from the "BACS-1" artificial character set ${ }^{158}$. This distraction task took approximately 5 minutes to complete and also provided a measure of unbiased working memory, which we calculated as the number of cards placed on the grid that matched the positions displayed (regardless of the symbol), plus the number of cards placed on the grid that matched both the positions and symbols displayed, averaged across all trials (equivalent to the Position Learning Index, or "PLI" score, of ${ }^{157}$ ).

Once this task had been completed, participants recorded their oral recollection of the creation story. They were given the opportunity to pause and continue recording, but were not allowed to return or re-record after advancing to the next task. This process, including the working memory distraction task, was then repeated for the second story and with the accent of opposite prestige level. Story order and accent were both randomized for presentation in the experiment. Each participant heard "Muki" in one accent condition and "Taka \& Toro" in the alternate accent condition.

After recording their recollections of both stories, participants listened to recordings of the Comma Gets a Cure passage read by the speakers providing the stories. To test that the different accents consistently indexed the expected differences in prestige, participants rated the speakers using the items for the Position-Reputation-Information (PRI) scale of individual prestige ${ }^{56}$ as well as additional solidarity and dynamism domains ${ }^{66}$. Finally, participants completed a demographic questionnaire including participants' residence history and self-reported accents of English. We collected data pertaining to gender and ethnicity in line with ethical practices in research and guidelines from national statistical agencies.

Data coding and transcription. We transcribed the audio files containing participants' story recordings, and coded each for the presence or absence of each proposition from the original texts (see Repository for coding protocols, transcripts and coded data). Because we instructed participants that they did not need to recall the stories verbatim, we counted the presence of a proposition if the meaning remained consistent through different word choices or constructions (e.g. we accepted synonyms and did not penalize the order of recall). If an error in the retellings was carried forward in the story, we only marked it absent in the first instance. We only counted biased propositions as present if the retelling retained the biased element (e.g. social interaction, counterintuitive properties, etc.).

To assess intercoder reliability, a second researcher re-coded a subset of 33 recordings (representing approximately $10 \%$ of the sample). We found substantial agreement between the coders (Cohen's $\mathrm{K}=0.737, p<0.01$ ), and coders discussed any disagreements until reaching consensus on the final coded data.

Data analysis. We used a set of generalized linear mixed models (GLMMs) to model the presence or absence of a particular proposition. Here, we tested the effects of eight different transmission biases by fitting a set of 58 candidate models that account for the potential effects of these biases in isolation and in combination with one another (Supplementary Table 3). For these models, the fixed effects we examined can be broken down into three categories of: 1) story- 
based effects (story, presentation order, and line number representing position in the story and quadratic line number representing primacy or recency effects); 2) transmission biases (prestige, social, survival, positive emotional, negative emotional, moral, rational, and counterintuitive domain); and 3) demographic effects (country, gender, ethnicity, accent matching low-prestige speaker, childhood town size, childhood town matching region of low-prestige speaker, education, occupation, income, and working memory score). Age was excluded from the demographic variables because of a lack of any predictive theory for its effects on recall beyond those of working memory. We also included random effects for participant and proposition number in all models to capture the remaining variance from these sources. Variable descriptions and the full data set are available in the Repository.

After model fitting, we compared models on the basis of each model's Akaike information criterion (AIC) score. Due to the lack of a single dominant model with a weight greater than 0.95 , we averaged the parameters of all models according to their Akaike weights ${ }^{159}$. As our main interest was in determining which factors had the strongest effects ${ }^{160}$, we determined full modelaveraged parameter estimates using the "zero method"159,161. This substitutes a value of zero for parameter estimates and errors in models where the parameter does not appear and computes a weighted average for each parameter using the models' Akaike weights.

We re-fit the full set of models using a continuous measure of the participants' perceptions of the speaker's prestige-as factor scores from the PRI scale of individual prestige ${ }^{56}$-rather than the binary high-low prestige variable, for the subset of participants that provided this information (roughly two thirds of the full data set). Results were qualitatively similar; however, direct comparisons cannot be made due to these analyses being performed on a nonrandom subset of the data.

We used the R statistical environment, versions 3.5.1 (2018-07-02) and 3.6.1 (2019-0705), for all analyses ${ }^{162}$.

Ethics statement. Prior approval for research protocols was obtained from the Colorado State University Institutional Review Board (protocol \#014-16H) and the University of Bristol Faculty of Arts Human Research Ethics Committee (protocols \#26561, \#31041, and \#38323). We obtained prior informed consent from all participants (full text of the consent form available at: https://github.com/seannyD/StoryTransmission/blob/master/www/StoryTransmission/survey/SUR VEY_consent.js). We compensated all participants for their time at rates above local minimum wages based upon the time taken to complete the tasks.

Participant data were gathered via a website hosted by the University of Bristol. A HyperText Transfer Protocol Secure (HTTPS) site was used to send data via an encrypted Transport Security Layer (TSL) to a University of Bristol virtual server that could only be accessed by the research team. Participants were assigned a random unique ID and no directly identifying information was gathered from participants. Amazon Mechanical Turk Worker IDs were encrypted and anonymized through TurkPrime to prevent identifiability. Voice recordings were securely stored in memory on the client side before being compressed and transferred to the virtual server via the encrypted TSL. Data were downloaded from the server to the local computers of the researchers for analysis via encrypted Secure Copy Protocol (SCP).

Data availability. All data and R scripts used for analysis will be deposited in a public repository prior to publication.

\section{Acknowledgments}


We thank Russell Gray for his feedback and advisement over the course of this project, the Max Planck Institute for the Science of Human History for funding, our participants and recorded speakers for their contributions, Rachel Sheer for authoring of the artificial creation stories, and the Native American Cultural Center at Colorado State University for their guidance and experience in navigating issues of cultural appropriation in the use of traditional stories. SGR was supported by a Leverhulme Early Career Fellowship (ECF-2016-435).

Comma Gets a Cure is copyright 2000 Douglas N. Honorof, Jill McCullough \& Barbara Somerville, text available online at: https://www.dialectsarchive.com/comma-gets-a-cure. Color palettes used in figures are derived from a technical note by Paul Tol (available at: https://personal.sron.nl/ pault/data/colourschemes.pdf) and are optimized for color-blind readers.

\section{References}

1. Bruner, J. S. The narrative construction of reality. Crit. Inq. 18, 1-21 (1991).

2. Bruner, J. S. Actual minds, possible worlds. (Harvard University Press, 2009).

3. Vansina, J. M. Oral tradition as history. (Univ of Wisconsin Press, 1985).

4. Lejano, R. P., Tavares-Reager, J. \& Berkes, F. Climate and narrative: Environmental knowledge in everyday life. Environ. Sci. Policy 31, 61-70 (2013).

5. Cajete, G. Look to the mountain: An ecology of indigenous education. (ERIC, 1994).

6. Piquemal, N. From Native North American oral traditions to Western literacy: Storytelling in education. Alta. J. Educ. Res. 49, (2003).

7. Chang, C. 'Being Hooked' By Editorial Content: The Implications for Processing Narrative Advertising. J. Advert. 38, 21-34 (2009).

8. Delgadillo, Y. \& Escalas, J. E. Narrative word-of-mouth communication: Exploring memory and attitude effects of consumer storytelling. Adv. Consum. Res. 31, 186-192 (2004).

9. White, M., White, M. K., Wijaya, M. \& Epston, D. Narrative means to therapeutic ends. (WW Norton \& Company, 1990).

10. Struthers, R., Eschiti, V. S. \& Patchell, B. Traditional indigenous healing: Part I. Complement. Ther. Nurs. Midwifery 10, 141-149 (2004).

11. Nunn, P. D. \& Reid, N. J. Aboriginal Memories of Inundation of the Australian Coast Dating from More than 7000 Years Ago. Aust. Geogr. 47, 11-47 (2016).

12. da Silva, S. G. \& Tehrani, J. J. Comparative phylogenetic analyses uncover the ancient roots of Indo-European folktales. R. Soc. Open Sci. 3, 150645 (2016).

13. Tehrani, J. J. \& d'Huy, J. Phylogenetics meets folklore: Bioinformatics approaches to the study of international folktales. in Maths Meets Myths: Quantitative Approaches to Ancient Narratives 91-114 (Springer, 2017).

14. Scalise Sugiyama, M. On the origins of narrative: Storyteller bias as a fitness-enhancing strategy. Hum. Nat. 7, 403-425 (1996).

15. Smith, D. et al. Cooperation and the evolution of hunter-gatherer storytelling. Nat. Commun. 8, (2017).

16. Boyd, B. On the origin of stories. (Harvard University Press, 2009).

17. Barrett, J. L. \& Nyhof, M. A. Spreading non-natural concepts: The role of intuitive conceptual structures in memory and transmission of cultural materials. J. Cogn. Cult. 1, 69-100 (2001).

18. Bebbington, K., MacLeod, C., Ellison, T. M. \& Fay, N. The sky is falling: evidence of a negativity bias in the social transmission of information. Evol. Hum. Behav. 38, 92-101 (2017).

19. Heath, C., Bell, C. \& Sternberg, E. Emotional selection in memes: The case of urban legends. J. Pers. Soc. Psychol. 81, 1028 (2001). 
20. Mesoudi, A., Whiten, A. \& Dunbar, R. A bias for social information in human cultural transmission. Br. J. Psychol. 97, 405-423 (2006).

21. Stubbersfield, J. M., Tehrani, J. J. \& Flynn, E. G. Serial killers, spiders and cybersex: Social and survival information bias in the transmission of urban legends. Br. J. Psychol. 288-307 (2015) doi:10.1111/bjop.12073.

22. Stubbersfield, J. M. \& Tehrani, J. Expect the unexpected? Testing for minimally counterintuitive $(\mathrm{MCl})$ bias in the transmission of contemporary legends: A computational phylogenetic approach. Soc. Sci. Comput. Rev. 31, 90-102 (2013).

23. Henrich, J., Boyd, R. \& Richerson, P. J. Five misunderstandings about cultural evolution. Hum. Nat. 19, 119-137 (2008).

24. Claidière, N., Scott-Phillips, T. C. \& Sperber, D. How Darwinian is cultural evolution? Philos. Trans. R. Soc. B Biol. Sci. 369, 20130368 (2014).

25. Acerbi, A. \& Mesoudi, A. If we are all cultural Darwinians what's the fuss about? Clarifying recent disagreements in the field of cultural evolution. Biol. Philos. 30, 481-503 (2015).

26. Morin, O. Reasons to be fussy about cultural evolution. Biol. Philos. 31, 447-458 (2016).

27. Cavalli-Sforza, L. L. \& Feldman, M. W. Cultural transmission and evolution: A quantitative approach. (Princeton University Press, 1981).

28. Boyd, R. \& Richerson, P. J. Culture and the evolutionary process. (University of Chicago Press, 1985).

29. Henrich, J. Cultural transmission and the diffusion of innovations: Adoption dynamics indicate that biased cultural transmission is the predominate force in behavioral change. Am. Anthropol. 103, 992-1013 (2001).

30. Rogers, A. R. Does biology constrain culture? Am. Anthropol. 90, 819-831 (1988).

31. Enquist, M., Eriksson, K. \& Ghirlanda, S. Critical social learning: A solution to Rogers's paradox of nonadaptive culture. Am. Anthropol. 109, 727-734 (2007).

32. Rendell, L., Fogarty, L. \& Laland, K. N. Rogers' paradox recast and resolved: Population structure and the evolution of social learning strategies. Evolution 64, 534-548 (2009).

33. McElreath, R. et al. Beyond existence and aiming outside the laboratory: Estimating frequency-dependent and pay-off-biased social learning strategies. Philos. Trans. R. Soc. $B$ Biol. Sci. 363, 3515-3528 (2008).

34. Kendal, R. L. et al. Social Learning Strategies: Bridge-Building between Fields. Trends Cogn. Sci. 22, 651-665 (2018).

35. Jiménez, Á. V. \& Mesoudi, A. Prestige-biased social learning: Current evidence and outstanding questions. Palgrave Commun. 5, 20 (2019).

36. Atkisson, C., Mesoudi, A. \& O'Brien, M. J. Adult learners in a novel environment use prestige-biased social learning. Evol. Psychol. Int. J. Evol. Approaches Psychol. Behav. 10, 519-537 (2012).

37. Morgan, T. J. H., Rendell, L., Ehn, M., Hoppitt, W. J. E. \& Laland, K. N. The evolutionary basis of human social learning. Proc. R. Soc. B Biol. Sci. 279, 653-662 (2012).

38. Acerbi, A. \& Tehrani, J. Did Einstein really say that? Testing content versus context in the cultural selection of quotations. J. Cogn. Cult. (2018).

39. Boyer, P. \& Ramble, C. Cognitive templates for religious concepts: Cross-cultural evidence for recall of counter-intuitive representations. Cogn. Sci. 25, 535-564 (2001).

40. Eriksson, K. \& Coultas, J. C. Corpses, maggots, poodles and rats: Emotional selection operating in three phases of cultural transmission of urban legends. J. Cogn. Cult. 14, 1-26 (2014).

41. Nairne, J. S., Thompson, S. R. \& Pandeirada, J. N. Adaptive memory: survival processing enhances retention. J. Exp. Psychol. Learn. Mem. Cogn. 33, 263 (2007).

42. Norenzayan, A. \& Atran, S. Cognitive and emotional processes in the cultural transmission of natural and nonnatural beliefs. Psychol. Found. Cult. 149-169 (2004). 
43. Barrett, H. C. \& Broesch, J. Prepared social learning about dangerous animals in children. Evol. Hum. Behav. 33, 499-508 (2012).

44. Otgaar, H. \& Smeets, T. Adaptive memory: Survival processing increases both true and false memory in adults and children. J. Exp. Psychol. Learn. Mem. Cogn. 36, 1010 (2010).

45. Fessler, D. M., Pisor, A. C. \& Navarrete, C. D. Negatively-biased credulity and the cultural evolution of beliefs. PLOS ONE 9, e95167 (2014).

46. Stubbersfield, J. M., Tehrani, J. J. \& Flynn, E. G. Chicken tumours and a fishy revenge: Evidence for emotional content bias in the cumulative recall of urban legends. J. Cogn. Cult. 17, 12-26 (2017).

47. Baumard, N. \& Boyer, P. Explaining moral religions. Trends Cogn. Sci. 17, 272-280 (2013).

48. Glenn, C. G. Relationship between story content and structure. J. Educ. Psychol. 72, 550 (1980).

49. Barrett, J. L. Coding and quantifying counterintuitiveness in religious concepts: Theoretical and methodological reflections. Method Theory Study Relig. 20, 308-338 (2008).

50. Norenzayan, A., Atran, S., Faulkner, J. \& Schaller, M. Memory and mystery: The cultural selection of minimally counterintuitive narratives. Cogn. Sci. 30, 531-553 (2006).

51. Henrich, J. \& Gil-White, F. J. The evolution of prestige: Freely conferred deference as a mechanism for enhancing the benefits of cultural transmission. Evol. Hum. Behav. 22, 165196 (2001).

52. Mesoudi, A. An experimental simulation of the "copy-successful-individuals" cultural learning strategy: adaptive landscapes, producer-scrounger dynamics, and informational access costs. Evol. Hum. Behav. 29, 350-363 (2008).

53. McElreath, R., Boyd, R. \& Richerson, P. J. Shared norms and the evolution of ethnic markers. Curr. Anthropol. 44, 122-130 (2003).

54. Mahajan, N. \& Wynn, K. Origins of "us" versus "them": Prelinguistic infants prefer similar others. Cognition 124, 227-233 (2012).

55. Henrich, J. \& Boyd, R. The evolution of conformist transmission and the emergence of between-group differences. Evol. Hum. Behav. 19, 215-241 (1998).

56. Berl, R. E. W., Samarasinghe, A. N., Jordan, F. M. \& Gavin, M. C. The Position-ReputationInformation (PRI) scale of individual prestige. PLOS ONE 15, e0234428 (2020).

57. Henrich, J. \& Boyd, R. On modeling cognition and culture. J. Cogn. Cult. 2, 87-112 (2002).

58. Henrich, J., Chudek, M. \& Boyd, R. The Big Man Mechanism: How prestige fosters cooperation and creates prosocial leaders. Philos. Trans. R. Soc. B 370, (2015).

59. Reyes-García, V. et al. Do the aged and knowledgeable men enjoy more prestige? A test of predictions from the prestige-bias model of cultural transmission. Evol. Hum. Behav. 29, 275-281 (2008).

60. Chudek, M., Baron, A. S. \& Birch, S. Unselective overimitators: The evolutionary implications of children's indiscriminate copying of successful and prestigious models. Child Dev. 87, 782-794 (2016).

61. Garfield, Z. H., Hubbard, R. L. \& Hagen, E. H. Evolutionary models of leadership. Hum. Nat. (2019) doi:10.1007/s12110-019-09338-4.

62. Labov, W. The social stratification of English in New York City. (Center for Applied Linguistics, 1966).

63. Giles, H. Evaluative Reactions to Accents. Educ. Rev. 22, 211-227 (1970).

64. Bishop, H., Coupland, N. \& Garrett, P. Conceptual accent evaluation: Thirty years of accent prejudice in the UK. Acta Linguist. Hafniensia 37, 131-154 (2005).

65. Coupland, N. \& Bishop, H. Ideologised values for British accents. J. Socioling. 11, 74-93 (2007). 
66. Fuertes, J. N., Gottdiener, W. H., Martin, H., Gilbert, T. C. \& Giles, H. A meta-analysis of the effects of speakers' accents on interpersonal evaluations: Effects of speakers' accents. Eur. J. Soc. Psychol. 42, 120-133 (2012).

67. Samarasinghe, A. N., Berl, R. E. W., Gavin, M. C. \& Jordan, F. M. Evaluations of accents can be used as a measure of prestige. SocArXiv (2019) doi:10.31235/osf.io/abgue.

68. Cronk, L. The application of animal signaling theory to human phenomena: Some thoughts and clarifications. Soc. Sci. Inf. 44, 603-620 (2005).

69. Kahane, H. A typology of the prestige language. Language 62, 495-508 (1986).

70. Kroch, A. S. Toward a theory of social dialect variation. Lang. Soc. 7, 17-36 (1978).

71. Kraus, M. W., Torrez, B., Park, J. W. \& Ghayebi, F. Evidence for the reproduction of social class in brief speech. Proc. Natl. Acad. Sci. 116, 22998-23003 (2019).

72. Ohlsen, G., van Zoest, W. \& van Vugt, M. Gender and facial dominance in gaze cuing: Emotional context matters in the eyes that we follow. PloS One 8, e59471 (2013).

73. Barkow, J. H. Prestige and the ongoing process of culture revision. in The Psychology of Social Status 29-45 (Springer, 2014).

74. Roberts, A., Palermo, R. \& Visser, T. A. Effects of dominance and prestige based social status on competition for attentional resources. Sci. Rep. 9, 2473 (2019).

75. Henrich, J. \& McElreath, R. The evolution of cultural evolution. Evol. Anthropol. 12, 123135 (2003).

76. Richerson, P. J. \& Boyd, R. Not by genes alone: How culture transformed human evolution. (University of Chicago Press, 2005).

77. Tamariz, M., Gong, T. \& Jäger, G. Investigating the effects of prestige on the diffusion of linguistic variants. in Annual Meeting of the Cognitive Science Society, CogSci 2011 (Cognitive Science Society. The Proceedings' web site is located at http://mindmodeling. org/cogsci2011/, 2011).

78. Chudek, M., Heller, S., Birch, S. \& Henrich, J. Prestige-biased cultural learning: Bystander's differential attention to potential models influences children's learning. Evol. Hum. Behav. 33, 46-56 (2012).

79. Cheng, J. T., Tracy, J. L., Foulsham, T., Kingstone, A. \& Henrich, J. Two ways to the top: Evidence that dominance and prestige are distinct yet viable avenues to social rank and influence. J. Pers. Soc. Psychol. 104, 103-125 (2013).

80. Boyd, R. \& Richerson, P. J. Culture and the evolution of human cooperation. Philos. Trans. R. Soc. B Biol. Sci. 364, 3281-3288 (2009).

81. Boyd, R., Richerson, P. J. \& Henrich, J. Rapid cultural adaptation can facilitate the evolution of large-scale cooperation. Behav. Ecol. Sociobiol. 65, 431-444 (2011).

82. Halevy, N., Chou, E. Y., Cohen, T. R. \& Livingston, R. W. Status conferral in intergroup social dilemmas: Behavioral antecedents and consequences of prestige and dominance. $J$. Pers. Soc. Psychol. 102, 351 (2012).

83. Rendell, L. et al. Cognitive culture: Theoretical and empirical insights into social learning strategies. Trends Cogn. Sci. 15, 68-76 (2011).

84. Dunbar, R. Theory of mind and the evolution of language. in Approaches to the Evolution of Language (eds. Hurford, J. R., Studdert-Kennedy, M. \& Knight, C.) 92-110 (Cambridge University Press, 1998).

85. Dunbar, R. I. M. Gossip in evolutionary perspective. Rev. Gen. Psychol. 8, 100-110 (2004).

86. Nairne, J. S. \& Pandeirada, J. N. Adaptive memory: Ancestral priorities and the mnemonic value of survival processing. Cognit. Psychol. 61, 1-22 (2010).

87. Kensinger, E. A. \& Corkin, S. Memory enhancement for emotional words: Are emotional words more vividly remembered than neutral words? Mem. Cognit. 31, 1169-1180 (2003).

88. Al-Shawaf, L., Conroy-Beam, D., Asao, K. \& Buss, D. M. Human Emotions: An Evolutionary Psychological Perspective. Emot. Rev. 8, 173-186 (2016). 
89. Al-Shawaf, L. \& Lewis, D. M. G. Evolutionary psychology and the emotions. in Encyclopedia of Personality and Individual Differences (eds. Zeigler-Hill, V. \& Shackelford, T. K.) 1-10 (Springer International Publishing, 2017). doi:10.1007/978-3-319-28099-8_516-1.

90. Barrett, H. C. Adaptations to Predators and Prey. in The Handbook of Evolutionary Psychology 1-18 (American Cancer Society, 2015). doi:10.1002/9781119125563.evpsych109.

91. Boyer, P. \& Barrett, H. C. Domain Specificity and Intuitive Ontology. in The Handbook of Evolutionary Psychology (ed. Buss, D. M.) 96-118 (John Wiley \& Sons, Inc., 2015). doi:10.1002/9780470939376.ch3.

92. Tooby, J. \& Cosmides, L. The Evolutionary Psychology of the Emotions and Their Relationship to Internal Regulatory Variables. in Handbook of Emotions (eds. Lewis, M., Haviland-Jones, J. M. \& Barrett, L. F.) 114-137 (Guilford Press, 2008).

93. Bartlett, F. C. Some experiments on the reproduction of folk-stories. Folklore 31, 30-47 (1920).

94. Horner, V. \& Whiten, A. Causal knowledge and imitation/emulation switching in chimpanzees (Pan troglodytes) and children (Homo sapiens). Anim. Cogn. 8, 164-181 (2005).

95. Buchsbaum, D., Gopnik, A., Griffiths, T. L. \& Shafto, P. Children's imitation of causal action sequences is influenced by statistical and pedagogical evidence. Cognition 120, 331-340 (2011).

96. Berl, R. E. W. \& Hewlett, B. S. Cultural variation in the use of overimitation by the Aka and Ngandu of the Congo Basin. PLOS ONE (2015) doi:10.1371/journal.pone.0120180.

97. Bender, A. \& Beller, S. The cultural fabric of human causal cognition. Perspect. Psychol. Sci. 14, 922-940 (2019).

98. Cialdini, R. B. \& Trost, M. R. Social influence: Social norms, conformity, and compliance. in The Handbook of Social Psychology (eds. Gilbert, D. T., Fiske, S. T. \& Lindzey, G.) 151192 (McGraw-Hill, 1998).

99. McDonald, R. I. \& Crandall, C. S. Social norms and social influence. Curr. Opin. Behav. Sci. 3, 147-151 (2015).

100. Jakobson, R. Linguistics and Poetics. in Style in Language 350-377 (MIT Press, 1960).

101. Waugh, L. R. The Poetic Function in the Theory of Roman Jakobson. Poet. Today 2, 57-82 (1980).

102. Literary theory: an anthology. (Blackwell Pub, 2004).

103. Busselle, R. \& Bilandzic, H. Measuring Narrative Engagement. Media Psychol. 12, 321-347 (2009).

104. Duranti, A. The audience as co-author: An introduction. Text 6, 239-247 (1986).

105. Graesser, A. C., Olde, B. \& Klettke, B. How does the mind construct and represent stories? in Narrative Impact: Social and Cognitive Foundations (eds. Green, M. C., Strange, J. J. \& Brock, T. C.) 229-262 (Lawrence Erlbaum, 2002).

106. Graesser, A. C., McNamara, D. S. \& Louwerse, M. M. What do readers need to learn in order to process coherence relations in narrative and expository text? in Rethinking Reading Comprehension (eds. Sweet, A. P. \& Snow, C. E.) 82-98 (Guilford Press, 2003).

107. Kormos, J. \& Trebits, A. Chapter 10 . Working memory capacity and narrative task performance. in Task-Based Language Teaching (ed. Robinson, P.) vol. 2 267-286 (John Benjamins Publishing Company, 2011).

108. Montgomery, J. W., Polunenko, A. \& Marinellie, S. A. Role of working memory in children's understanding spoken narrative: A preliminary investigation. Appl. Psycholinguist. 30, 485 (2009). 
768

769

109. Ward, C. M., Rogers, C. S., Van Engen, K. J. \& Peelle, J. E. Effects of Age, Acoustic Challenge, and Verbal Working Memory on Recall of Narrative Speech. Exp. Aging Res. 42, 97-111 (2016).

110. Andringa, E. Effects of 'narrative distance' on readers' emotional involvement and response. Poetics 23, 431-452 (1996).

111. Hänninen, K. Perspectives on the narrative construction of emotions. Elore 14, 1-9 (2007).

112. Komeda, H., Kawasaki, M., Tsunemi, K. \& Kusumi, T. Differences between estimating protagonists' emotions and evaluating readers' emotions in narrative comprehension. Cogn. Emot. 23, 135-151 (2009).

113. Benelli, E. et al. Emotional and cognitive processing of narratives and individual appraisal styles: recruitment of cognitive control networks vs. modulation of deactivations. Front. Hum. Neurosci. 6, (2012).

114. Genter, D. R. The structure and recall of narrative prose. J. Verbal Learn. Verbal Behav. 15, 411-418 (1976).

115. Thorndyke, P. W. Cognitive Structures in Comprehension and Memory of Narrative Discourse.pdf. Cognit. Psychol. 9, 77-110 (1977).

116. Efferson, C., Lalive, R., Richerson, P. J., McElreath, R. \& Lubell, M. Conformists and mavericks: the empirics of frequency-dependent cultural transmission. Evol. Hum. Behav. 29, 56-64 (2008).

117. Baldini, R. Success-biased social learning: Cultural and evolutionary dynamics. Theor. Popul. Biol. 82, 222-228 (2012).

118. Efferson, C. et al. Learning, productivity, and noise: An experimental study of cultural transmission on the Bolivian Altiplano. Evol. Hum. Behav. 28, 11-17 (2007).

119. Strimling, P., Enquist, M. \& Eriksson, K. Repeated learning makes cultural evolution unique. Proc. Natl. Acad. Sci. 106, 13870-13874 (2009).

120. Tomasello, M., Kruger, A. C. \& Ratner, H. H. Cultural learning. Behav. Brain Sci. 4, 101143 (1993).

121. Tennie, C., Call, J. \& Tomasello, M. Ratcheting up the ratchet: On the evolution of cumulative culture. Philos. Trans. R. Soc. B Biol. Sci. 364, 2405 (2009).

122. Lewis, H. M. \& Laland, K. N. Transmission fidelity is the key to the build-up of cumulative culture. Philos. Trans. R. Soc. B Biol. Sci. 367, 2171-2180 (2012).

123. Dean, L. G., Vale, G. L., Laland, K. N., Flynn, E. \& Kendal, R. L. Human cumulative culture: A comparative perspective. Biol. Rev. 89, 284-301 (2014).

124. Caldwell, C. A., Renner, E. \& Atkinson, M. Human Teaching and Cumulative Cultural Evolution. Rev. Philos. Psychol. 9, 751-770 (2018).

125. Sperber, D. Explaining Culture: A Naturalistic Approach. (Blackwell Publishers Ltd., 1996).

126. Sasaki, T. \& Biro, D. Cumulative culture can emerge from collective intelligence in animal groups. Nat. Commun. 8, 15049 (2017).

127. Zwirner, E. \& Thornton, A. Cognitive requirements of cumulative culture: Teaching is useful but not essential. Sci. Rep. 5, 16781 (2015).

128. McElreath, M. B., Boesch, C., Kuehl, H. \& McElreath, R. Complex dynamics from simple cognition: The primary ratchet effect in animal culture. Evol. Behav. Sci. 12, 191 (2018).

129. Miton, H. \& Charbonneau, M. Cumulative culture in the laboratory: Methodological and theoretical challenges. Proc. R. Soc. B Biol. Sci. 285, 20180677 (2018).

130. Truskanov, N. \& Prat, Y. Cultural transmission in an ever-changing world: trial-and-error copying may be more robust than precise imitation. Philos. Trans. R. Soc. B Biol. Sci. 373, 20170050 (2018).

131. Miu, E., Gulley, N., Laland, K. N. \& Rendell, L. Innovation and cumulative culture through tweaks and leaps in online programming contests. Nat. Commun. 9, (2018). 
132. Kirby, S., Dowman, M. \& Griffiths, T. L. Innateness and culture in the evolution of language. Proc. Natl. Acad. Sci. 104, 5241-5245 (2007).

133. Thompson, B., Kirby, S. \& Smith, K. Culture shapes the evolution of cognition. Proc. Natl. Acad. Sci. 113, 4530-4535 (2016).

134. Dennis, G. \& Walter, E. The Effects of Repeated Read-Alouds on Story Comprehension as Assessed Through Story Retellings. Read. Improv. 32, 140 (1995).

135. Norrick, N. R. Conversational storytelling. in The Cambridge Companion to Narrative 127141 (2007).

136. Lawrence, D. \& Thomas, J. C. Social Dynamics of Storytelling: Implications for Story-Base Design. 4 (1999).

137. Norrick, N. R. Twice-told tales: Collaborative narration of familiar stories. Lang. Soc. 26 , 199-220 (1997).

138. Mesoudi, A., Chang, L., Murray, K. \& Lu, H. J. Higher frequency of social learning in China than in the West shows cultural variation in the dynamics of cultural evolution. Proc. R. Soc. B Biol. Sci. 282, 20142209-20142209 (2014).

139. Eriksson, K., Coultas, J. C. \& Barra, M. de. Cross-Cultural Differences in Emotional Selection on Transmission of Information. J. Cogn. Cult. 16, 122-143 (2016).

140. Leeuwen, E. J. et al. The development of human social learning across seven societies. Nat. Commun. 9, 2076 (2018).

141. Schwartz, T. \& Mead, M. Micro- and Macro-Cultural Models for Cultural Evolution. Anthropol. Linguist. 3, 1-7 (1961).

142. Kandler, A. \& Powell, A. Inferring learning strategies from cultural frequency data. in Learning strategies and cultural evolution during the Palaeolithic 85-101 (Springer, 2015).

143. Human Relations Area Files. eHRAF World Cultures Database. https://ehrafworldcultures.yale.edu.

144. The New Oxford Annotated Bible: New Revised Standard Version with the Apocrypha: An Ecumenical Study Bible. (Oxford University Press, 2010).

145. Turner, A. \& Greene, E. The construction and use of a propositional text base. (Institute for the Study of Intellectual Behavior, University of Colorado Boulder, Colorado, 1977).

146. Garrett, P. Language attitudes. in The Routledge Companion to Sociolinguistics 116 (2007).

147. Labov, W. Phonological correlates of social stratification. Am. Anthropol. 66, 164-176 (1964).

148. Labov, W. The Social Stratification of ( $r$ ) in New York City Department Stores. in Sociolinguistic Patterns 43-52 (University of Pennsylvania Press, 1972).

149. Giles, H. Communicative effectiveness as a function of accented speech. (1973).

150. The Ideology of the Standard Language. in The Routledge companion to sociolinguistics (ed. Milroy, J.) 133-139 (Routledge, 2007).

151. Milroy, J. \& Milroy, L. Authority in Language: Investigating Standard English. (Psychology Press, 1999).

152. Stewart, M. A., Ryan, E. B. \& Giles, H. Accent and social class effects on status and solidarity evaluations. Pers. Soc. Psychol. Bull. 11, 98-105 (1985).

153. Coupland, N. Sociolinguistic authenticities. J. Socioling. 7, 417-431 (2003).

154. Giles, H. Patterns of evaluation to RP, South Welsh and Somerset accented speech. Br. J. Soc. Clin. Psychol. 10, 280-281 (1971).

155. Wells, J. C. Accents of English. (Cambridge University Press, 1982).

156. Litman, L., Robinson, J. \& Abberbock, T. TurkPrime.com: A versatile crowdsourcing data acquisition platform for the behavioral sciences. Behav. Res. Methods 49, 433-442 (2017).

157. Malec, J. E., Ivnik, R. J. \& Hinkeldey, N. S. Visual Spatial Learning Test. Psychol. Assess. J. Consult. Clin. Psychol. 3, 82-88 (1991). 
158. Vidal, C., Content, A. \& Chetail, F. BACS: The Brussels Artificial Character Sets for studies in cognitive psychology and neuroscience. Behav. Res. Methods 49, 2093-2112 (2017). 159. Burnham, K. P. \& Anderson, D. R. Model selection and multimodel inference. (Springer, 2002).

822

823

160. Nakagawa, S. \& Freckleton, R. P. Model averaging, missing data and multiple imputation: a case study for behavioural ecology. Behav. Ecol. Sociobiol. 65, 103-116 (2011).

161. Grueber, C. E., Nakagawa, S., Laws, R. J. \& Jamieson, I. G. Multimodel inference in ecology and evolution: challenges and solutions: Multimodel inference. J. Evol. Biol. 24, 699-711 (2011).

162. R Core Team. R: A Language and Environment for Statistical Computing. (R Foundation for Statistical Computing (https://www.R-project.org/), 2018). 
Figures

833

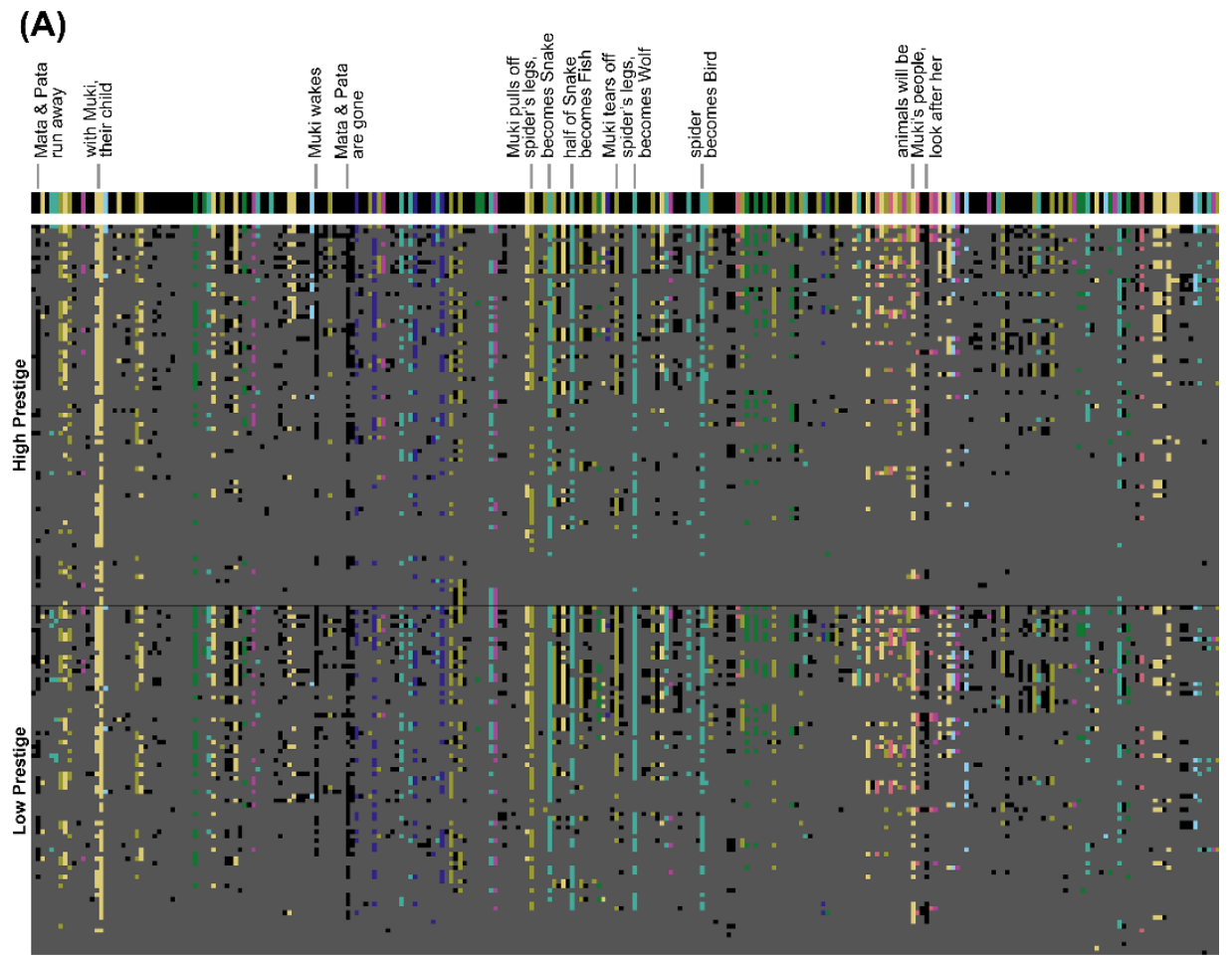

(B)
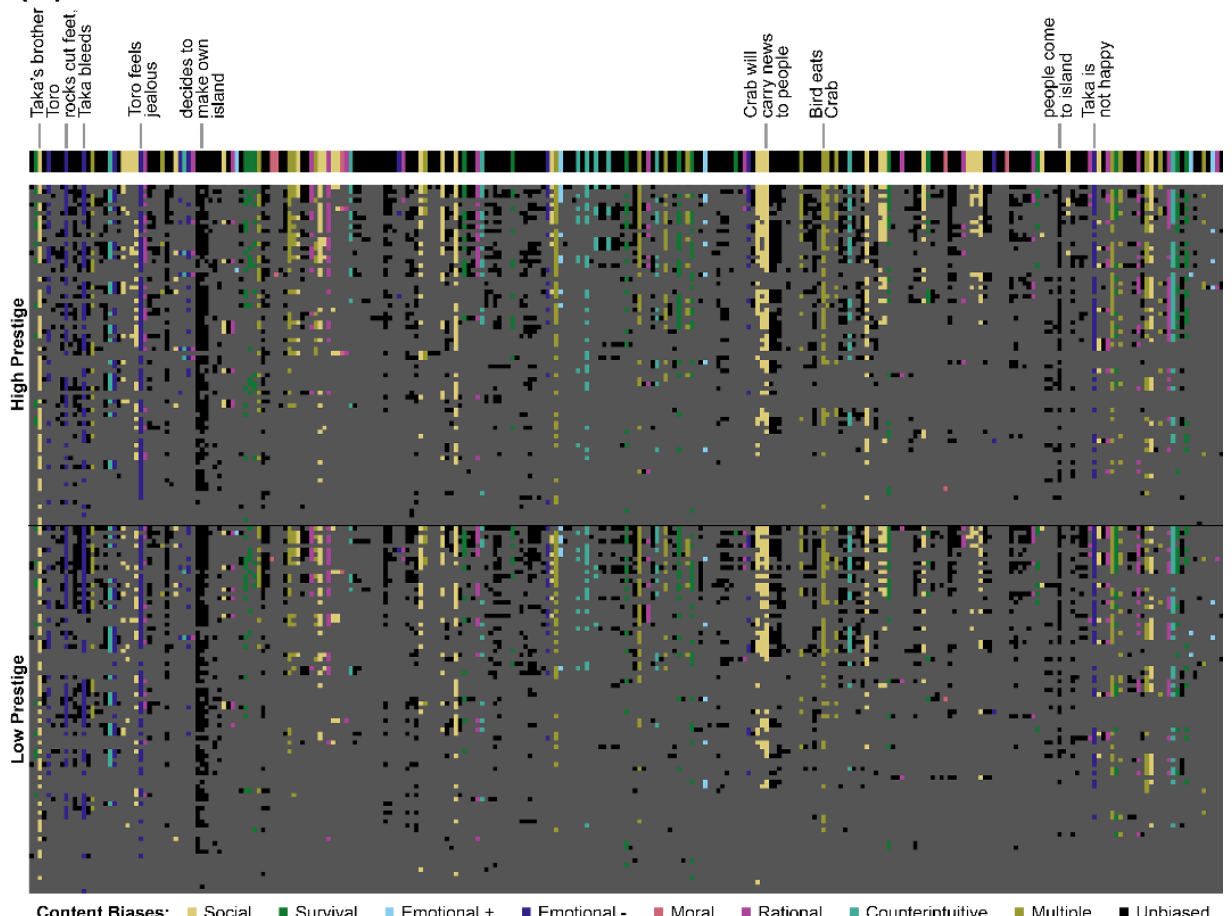
Fig. 1. Color matrices of the presence or absence of propositions in recalled stories. Each row represents one participant's recall $(n=165$ per panel), sorted by hierarchical clustering for visibility. Each column is a proposition from the Muki (A) or Taka \& Toro (B) artificial creation stories, from left to right in the order in which the propositions appeared in the stories. The thick line above each panel shows the full set of propositions contained in the story as originally told, with labels indicating propositions with exceptionally high recall (using Tukey's definition of outliers). Within each panel, rows in the upper portion were read by a high-prestige speaker, while rows in the lower portion were read by a low-prestige speaker. Dark gray propositions were not recalled (absent). Recalled propositions (present) are each represented by a color that indicates the content biases they contained, as indicated at the bottom of the figure: social information is yellow, survival is green, positive emotional is light blue, negative emotional is dark purple, moral is pink, rational is magenta, counterintuitive is teal, and propositions containing more than one bias are gold. Unbiased propositions, those that did not contain any biased 


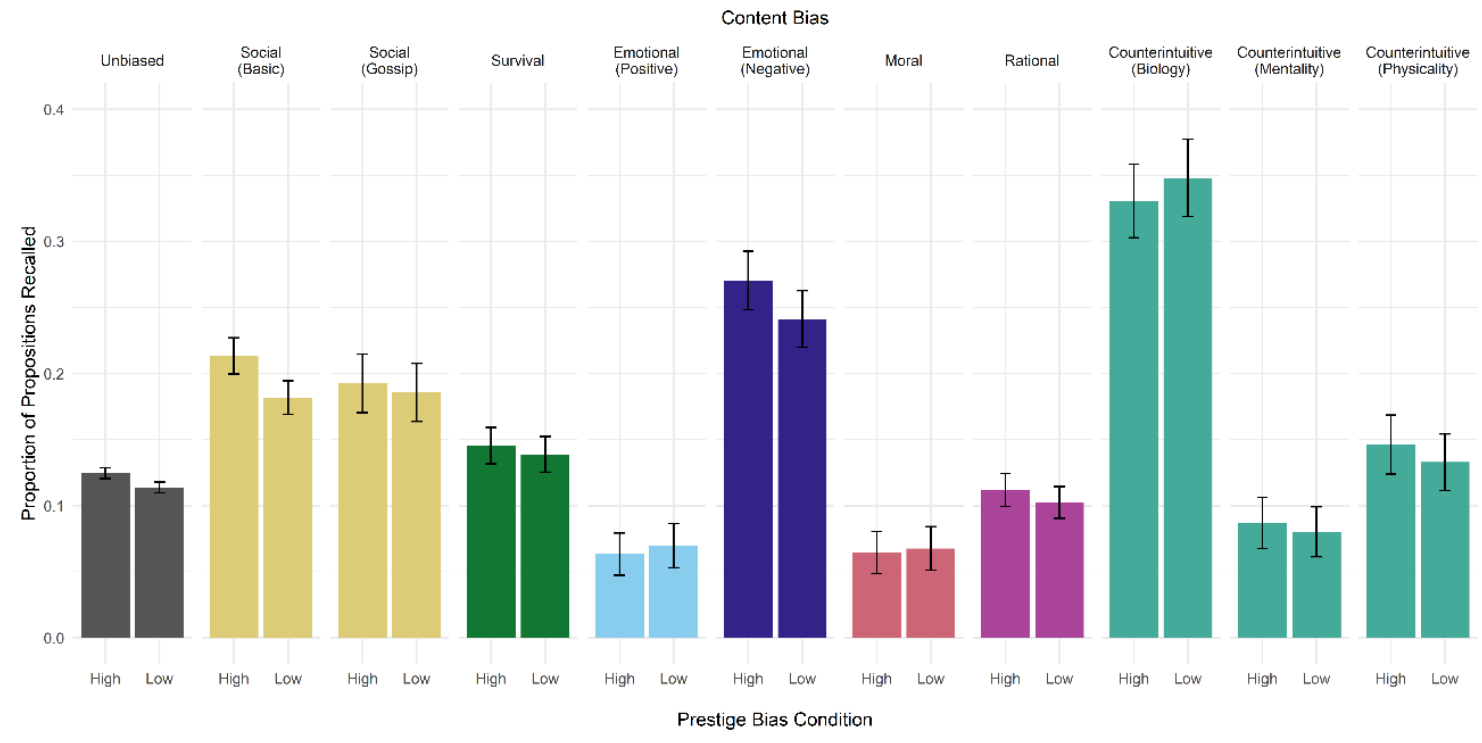

Fig. 2. Mean proportion of propositions recalled from artificial creation stories by type of content bias and by speaker prestige. Error bars represent 95\% confidence intervals. Propositions containing more than one type of content bias are excluded ( $N=10,864$ propositions recalled / 78,965 presented). 


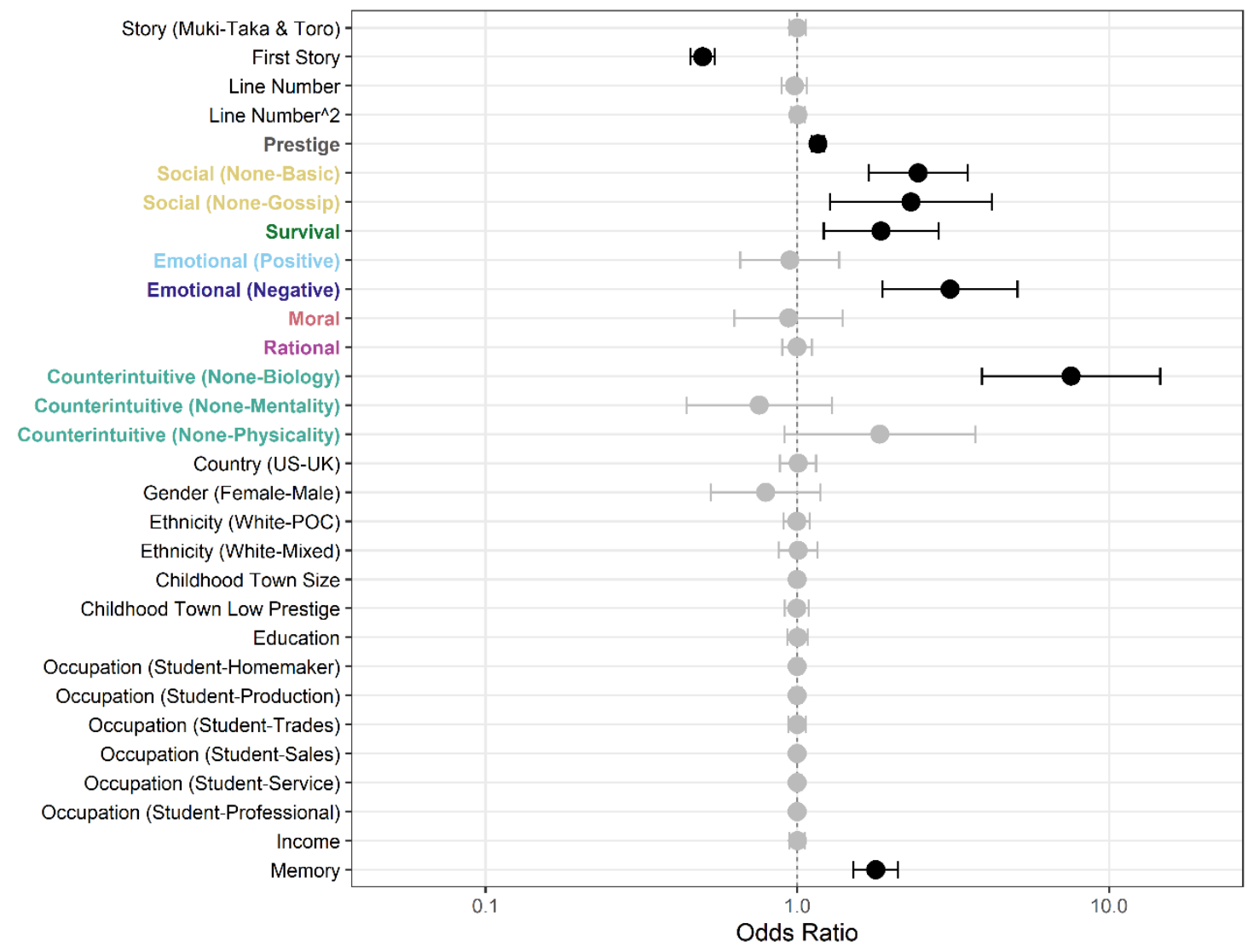

Fig. 3. Forest plot of odds ratios from full model-averaged coefficients for fixed effects. Odds ratios and $95 \%$ confidence intervals are depicted such that variables for which confidence intervals do not overlap with 1 have a significant positive (above 1) or negative (below 1) effect on proposition recall (black), compared to variables that did not have a significant effect (gray). Binary and categorical variables are represented relative to the reference level (false/not present unless specified otherwise). For ordinal variables (childhood town size, education, and income), only linear contrasts are shown. 